OPEN ACCESS

Check for updates

\title{
Association between human papillomavirus vaccination and serious adverse events in South Korean adolescent girls: nationwide cohort study
}

\author{
Dongwon Yoon, ${ }^{1}$ Ji-Ho Lee, ${ }^{1,2}$ Hyesung Lee, ${ }^{1}$ Ju-Young Shin ${ }^{1,3}$
}

${ }^{1}$ School of Pharmacy,

Sungkyunkwan University, 2066, Seobu-ro, Jangan-gu, Suwon, Gyeonggi-do, South Korea

${ }^{2}$ Chung-Ang University Hospital, 06973, 102 Heukseok-ro,

Dongjak-gu, Seoul, South Korea

${ }^{3}$ Samsung Advanced Institute for Health Sciences and Technology (SAIHST), Sungkyunkwan

University, Seoul, South Korea

Correspondence to: J-YShin

shin.jy@skku.edu

(ORCID 0000-0003-1010-7525)

Additional material is published online only. To view please visit the journal online.

Cite this as: $B M J$ 2021;372:m4931 http://dx.doi.org/10.1136/bmj.m493

Accepted: 2 December 2020

\section{ABSTRACT}

OBJECTIVE

To evaluate the association between human papillomavirus (HPV) vaccination and serious adverse events in adolescent girls in South Korea.

\section{DESIGN}

Cohort study.

SETTING

A large linked database created by linking the Korea Immunization Registry Information System and the National Health Information Database, between January 2017 and December 2019.

\section{PARTICIPANTS}

441399 girls aged 11-14 years who had been vaccinated in 2017: 382020 had been vaccinated against HPV and 59379 had not been vaccinated against HPV.

\section{MAIN OUTCOME MEASURES}

Outcomes were 33 serious adverse events, including endocrine, gastrointestinal, cardiovascular, musculoskeletal, haematological, dermatological, and neurological diseases. A cohort design was used for the primary analysis and a self-controlled risk interval design for the secondary analysis; both analyses used a risk period of one year after HPV vaccination for each outcome. Incidence rate and adjusted rate ratios were estimated using Poisson regression in the primary analysis, comparing the HPV vaccinated group with the HPV unvaccinated group, and adjusted relative risks were estimated using conditional logistic regression in the secondary analysis.

RESULTS

Among the 33 predefined serious adverse events, no associations were found with HPV vaccination in the cohort analysis, including Hashimoto's thyroiditis (incidence rate per 100000 person years: $52.7 v 36.3$ for the vaccinated and unvaccinated groups; adjusted

\section{WHAT IS ALREADY KNOWN ON THIS TOPIC}

Serious adverse events after immunisation with the human papillomavirus (HPV) vaccine are one of the major concerns and barriers to HPV vaccination

Although the real world evidence on the safety of the HPV vaccine is established for Western populations, evidence remains insufficient for Asian populations

\section{WHAT THIS STUDY ADDS}

This study found no associations between HPV vaccination and 33 predefined serious adverse events, using both cohort and self-controlled risk interval analyses

Our results are consistent with those of studies that have shown the safety of HPV vaccination in Western populations rate ratio $1.24,95 \%$ confidence interval 0.78 to 1.94 ) and rheumatoid arthritis (incidence rate per 100000 person years: $168.1 v 145.4$ for the vaccinated and unvaccinated groups; $0.99,0.79$ to 1.25 ), with the exception of an increased risk observed for migraine (incidence rate per 100000 person years: $1235.0 \mathrm{~V}$ 920.9 for the vaccinated and unvaccinated groups; $1.11,1.02$ to 1.22$)$. Secondary analysis using selfcontrolled risk intervals confirmed no associations between HPV vaccination and serious adverse events, including migraine (adjusted relative risk 0.67, 95\% confidence interval 0.58 to 0.78 ). Results were robust to varying follow-up periods and for vaccine subtypes.

\section{CONCLUSIONS}

In this nationwide cohort study, with more than 500000 doses of HPV vaccines, no evidence was found to support an association between HPV vaccination and serious adverse events using both cohort analysis and self-controlled risk interval analysis. Inconsistent findings for migraine should be interpreted with caution considering its pathophysiology and the population of interest.

\section{Introduction}

In 2012, about 630000 women received a diagnosis of human papillomavirus (HPV) related cancer, of whom 530000 (84\%) had cervical cancer. ${ }^{1}$ Around 266000 women with cervical cancer died in 2012, accounting for $8 \%$ of all cancer related mortality in women worldwide. ${ }^{2}$ To reduce the HPV attributable burden of disease, vaccines against HPV have been introduced globally and are indicated for both adolescent boys and adolescent girls. ${ }^{3}$ The current World Health Organization recommendation for HPV immunisation prioritises girls aged 9-14 years before they become sexually active, and in 2019, 124 countries adopted the policy of HPV vaccination as their national immunisation programme for adolescent girls. ${ }^{4} 5$ Since June 2016, the Korea Disease Control and Prevention Agency has also introduced a two dose schedule of bivalent or quadrivalent HPV vaccines for adolescent girls aged 12-13 years as part of its national immunisation programme.${ }^{67}$ Coverage of the first HPV vaccine dose in the first year of implementation was $61.5 \%$, and by 2018 this had increased to $87.2 \% .^{8}$

Despite the extensive use of the HPV vaccine worldwide, safety concerns about HPV vaccination have not diminished, and concerns about vaccine related serious adverse events have undermined public confidence about immunisation. ${ }^{9}{ }^{10}$ A survey among Korean parents who did not want their children to receive HPV vaccination indicated that $73.5 \%$ were 
reluctant because of concerns about serious adverse events. ${ }^{11}$ To alleviate these concerns, good quality evidence on the safety of the vaccine in the Korean population is needed. Because most studies have focused on Western populations, ${ }^{12-18}$ information on the safety profile of HPV vaccine in Asian populations is insufficient. Furthermore, previous studies compared participants who had been vaccinated with those who had not, which could lead to biased results because of fundamental differences in personal and clinical characteristics. Moreover, studies have focused on the bivalent or quadrivalent HPV vaccine and thus provide information on the safety of certain types of HPV vaccines. ${ }^{12} 1319$

Given the public concerns and limited information in the Korean population, we investigated the association between HPV vaccination and serious adverse events among adolescent girls in South Korea using a nationwide linked database.

\section{Methods}

Data sources

We combined data from the Korea Immunization Registry Information System and the National Health Information Database to create a large database between January 2017 and December 2019 of adolescent girls aged 11-14 years in 2017. Using deidentified personal codes, we retrieved data from the Korea Immunization Registry Information System on type of vaccine administered, date of vaccination, dosing schedule, coadministered vaccines, and age at vaccination, and data from the National Health Information Database on medical diagnosis and date of diagnosis, prescription, treatment, medical insurance type, residence type, and total income of the affiliated households. This large linked database enabled us to evaluate the association between HPV vaccination and serious adverse events using both immunisation and clinical information.

According to the system of universal insurance coverage in South Korea, the National Health Information Database represents the entire population (50 million). ${ }^{20}$ This database includes information on personal details and clinical records on reimbursed inpatient and outpatient visits. Diagnosis and drug prescription information was coded using ICD-10 (international classification of diseases, 10th revision) and Anatomical Therapeutic Chemical classification codes, respectively. The positive predictive value for diagnosis codes of claims data was reported to be $82 \%$ in a validation study. ${ }^{21}$

\section{Study population}

To control for the unmasking phenomenon and fundamental differences between vaccinated and unvaccinated individuals, ${ }^{22}$ we selected Japanese encephalitis vaccine and tetanus, diphtheria, and acellular pertussis vaccine as comparators in the cohort analysis as they have well established safety profiles and are recommended in the same population of interest. ${ }^{23-25}$ Hence, the study population comprised girls aged 11-14 years who were vaccinated against HPV, Japanese encephalitis, and tetanus, diphtheria, and acellular pertussis in 2017. We excluded girls who were immunised with both bivalent and quadrivalent HPV vaccines in 2017, as well as those with a diagnosis of malignancy or congenital or neonatal disease (congenital iodine deficiency syndrome, congenital hypothyroidism, transitory neonatal hyperthyroidism, other specified diseases of the pancreas, transient neonatal thrombocytopenia, neonatal diabetes mellitus, cerebral palsy, and type 1 diabetes mellitus) before the first date of vaccination.

\section{Vaccination}

In South Korea, healthcare providers report detailed information on immunisation to the Korea Immunization Registry Information System and the government reimburses immunisation fees for all vaccines covered by the national immunisation programme. We obtained data on HPV, Japanese encephalitis, and tetanus, diphtheria, and acellular pertussis vaccinations from the Korea Immunization Registry Information System. A total of 443290 adolescents girls received the study vaccines (HPV, Japanese encephalitis, and tetanus, diphtheria, and acellular pertussis) in 2017. We classified girls vaccinated with HPV as the HPV vaccinated group, and those vaccinated with comparator vaccines without HPV vaccination as the HPV unvaccinated group. Cohort entry was defined as the date of vaccination. Each girl could have two records of cohort entry because HPV vaccination comprises a two dosing schedule with an interval of six months between doses.

\section{Outcomes}

We selected 33 serious adverse events as the main outcomes, predefined based on literature review, including case reports, pharmacovigilance studies, observational studies, meta-analysis studies, and a review of the Korean medical advisory committee on vaccine safety. ${ }^{1314161826-32}$ Outcomes were classified into eight disease categories: endocrine (Graves' disease, Hashimoto's thyroiditis, hyperthyroidism, hypothyroidism, and type 1 diabetes), gastrointestinal (Crohn's disease, ulcerative colitis, peptic ulcer, and pancreatitis), cardiovascular (Raynaud's disease, venous thromboembolism, vasculitis, and hypotension), musculoskeletal and systemic (ankylosing spondylitis, Behcet's syndrome, juvenile arthritis, rheumatoid arthritis, and systemic lupus erythematosus), haematological (idiopathic thrombocytopenic purpura, and Henoch-Schönlein's purpura), dermatological (erythema nodosum and psoriasis), neurological (Bell's palsy, epilepsy, narcolepsy, paralysis, migraine, Guillain-Barré syndrome, optical neuritis, neuralgia and neuritis, intracerebral haemorrhage, and extrapyramidal and movement disorders), and tuberculosis. Incident serious adverse events were identified using diagnosis records coded by ICD-10 (supplementary table S1). 


\section{Potential confounders}

We included sociodemographic characteristics and anaemia as potential confounders in our analyses based on a causal diagram using a directed acyclic graph (supplementary figure S1). Because sociodemographic characteristics are well known confounders in vaccine safety studies, ${ }^{12}{ }^{13}$ we assessed age, region of residence, type of health insurance, and income level at cohort entry. In addition, because anaemia is related to HPV vaccination and could affect the health outcome of interest (supplementary section A), we assessed anaemia within one year before cohort entry.

\section{Statistical analyses}

To assess the association between HPV vaccination and the predefined serious adverse events separately we used both a cohort and a self-controlled risk interval analysis (fig 1). Sociodemographic, clinical, and vaccination related characteristics are summarised in our study population. In addition, we present the mean days (interquartile range) to disease diagnosis after each dose of HPV vaccine.

\section{Cohort analyses}

We used a cohort design for our primary analysis because the results are more generalisable than those of the self-controlled risk interval analysis when vaccinated groups are compared with unvaccinated groups. Moreover, use of a nationwide linked database enables the association between HPV vaccine and serious adverse events to be identified without loss of statistical power. We defined the follow-up period as one year (365 days) to detect a potential insidious outcome and a delayed onset to diagnosis. To include incident cases only, we excluded girls who experienced a serious adverse event within one year before cohort entry. In the cohort analysis, we used a Poisson regression model to estimate the incidence rate ratio with $95 \%$ confidence interval. The incidence rate was calculated by dividing the number of serious adverse events by the total person years at risk and then multiplying the estimates by 100000 . The model was adjusted for age, region of residence, type of health insurance, income level, and anaemia.

\section{Self-controlled risk interval analyses}

We used the self-controlled risk interval design for secondary analysis by including girls with a history of both HPV vaccination and predefined serious adverse events. A major benefit of this within person comparison is that time invariant confounders (sex, region of residence, type of health insurance, income level, lifestyle) are self-adjusted, and the design is less subject to unmeasured confounders compared with other study designs. Using the self-controlled risk interval design, we tried to minimise the impact of bias from exposure misclassification and unmeasured time invariant confounders, which are limitations of the cohort design. ${ }^{33}$ The risk interval was defined as one year (365 days) after HPV vaccination to allow for latent diseases, and the control interval was defined as 466-730 days after HPV vaccination. We adopted a wash-out period of 90 days between the risk interval and control interval considering the possibility of difference between the disease onset and date of disease diagnosis. ${ }^{34}$ Only incident cases were included, and girls with serious adverse events in the preceding one year were excluded. We used a conditional logistic regression model to estimate the relative risk with 95\% confidence interval for each serious adverse event. The model was adjusted for age, which is a time varying variable between the risk interval and the control interval.

Primary analysis: cohort design

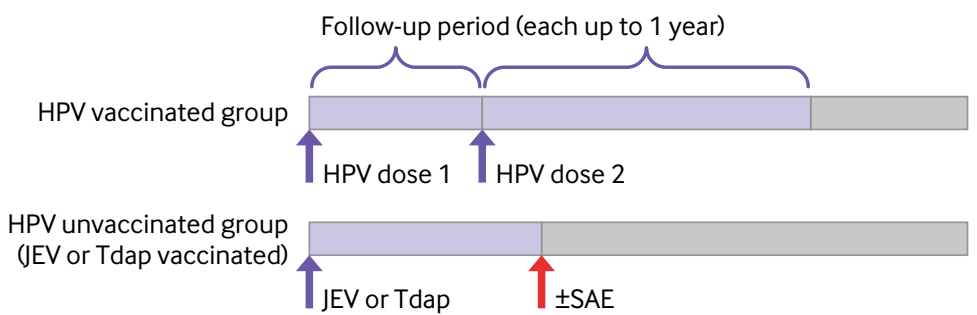

Secondary analysis: self-controlled risk interval design

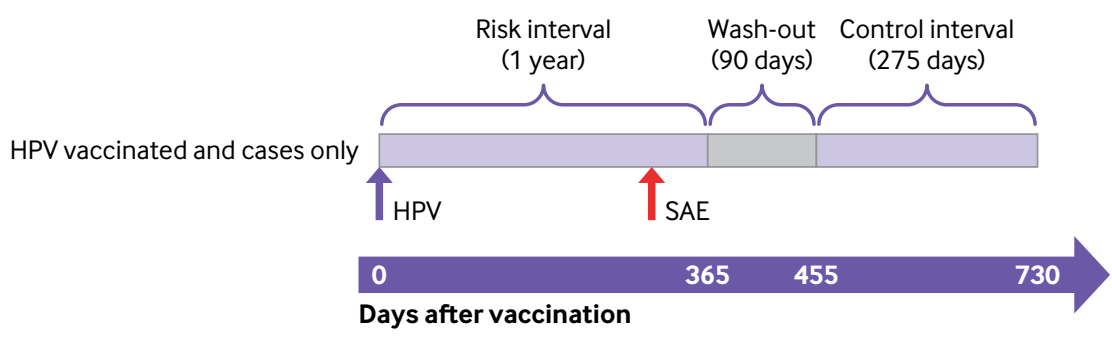

Fig 1 | Overview of cohort and self-controlled risk interval study designs. HPV=human papillomavirus; SAE=serious adverse event; JEV=Japanese encephalitis vaccine; Tdap=tetanus, diphtheria, and acellular pertussis vaccine 


\section{Sensitivity and subgroup analyses}

To confirm the robustness of the main analysis and to examine the possibility of an insidious outcome that was not identified even with a one year follow-up period, we varied the follow-up period in sensitivity analyses: short term (90 days and 180 days) and long term (two years (730 days)). We conducted subgroup analyses according to the subtypes of HPV (bivalent and quadrivalent) and then compared the HPV vaccinated group with the HPV unvaccinated groups to confirm the difference in risk among those factors.

All statistical analyses were performed using SAS Enterprise Guide 7.1 for Windows (SAS Institute, Cary, NC). A two tailed $P$ value of less than 0.05 was considered significant.

\section{Patient and public involvement}

As we used deidentified data in our analyses, the need for informed consent was waived, and the study was conducted in accordance with the Guidelines for Good Pharmacoepidemiology Practices. No patients were involved in establishing the research question or outcome measures or in developing plans for recruitment, design, or implementation of the study. No patients were asked for advice on interpretation or documentation of the results. We have no plans to disseminate the results of the research to study participants or the relevant patient community.

\section{Results}

Cohort

Of the total 676677 girls aged 11-14 years in South Korea, $441399(65 \%)$ were included in this study (fig 2). Among these girls, 382020 were inoculated with 429377 doses of the HPV vaccine and 59379 with 87099 doses of Japanese encephalitis vaccine or tetanus, diphtheria, and acellular pertussis vaccine without HPV vaccination (table 1 and fig 2). Among the HPV vaccinated girls, 295365 received the quadrivalent vaccine and 86655 received the bivalent vaccine (supplementary table S9). A total of 516476 HPV vaccine doses were administered to the study population. The mean follow-up time for each outcome in the HPV vaccinated and HPV unvaccinated groups was 407400 and 60500 person years, respectively (table 2).

Risk of serious adverse events after HPV vaccination The mean number of days to diagnosis of the 33 predefined outcomes after HPV vaccination was generally consistent with each other (supplementary table S2). Among the 33 predefined outcomes, the overall estimates in the primary analyses showed no association between HPV vaccines and serious adverse events (fig 3). Rate ratios could not be calculated for ankylosing spondylitis, systemic lupus erythematosus, and erythema nodosum because none occurred in the HPV unvaccinated group. The risk for type 1 diabetes was not significantly increased (adjusted rate ratio $0.66,95 \%$ confidence interval 0.38 to 1.14), and the result for the association between
HPV vaccination and Hashimoto's thyroiditis was not significant (1.24, 0.78 to 1.94$)$. Other conditions not associated with HPV vaccination were cardiovascular diseases, including Raynaud's disease (1.34, 0.40 to 4.49), and venous thromboembolism (0.62, 0.22 to 1.70). In musculoskeletal and systemic diseases, the rate ratio for the association between HPV vaccination and Behcet's syndrome was 0.61 (0.22 to 1.67). No significant increase in risk was found for haematological and dermatological diseases. Although neurological diseases, including epilepsy, narcolepsy, paralysis, and Guillain-Barré syndrome showed no significant increase in risk, a significant increase was observed for migraine. However, the lower limit of the $95 \%$ confidence interval was close to $1(1.11,1.02$ to 1.22). In line with the results of the cohort analysis, the results of the self-controlled risk interval analysis showed no significant increase in risk for all individual outcomes, including migraine (adjusted relative risk $0.67,95 \%$ confidence interval 0.58 to 0.78 ).

\section{Sensitivity and subgroup analyses}

Results of the sensitivity analyses with diverse followup periods (90 days, 180 days, and 2 years) were consistent with those of the main findings, which showed no association between HPV vaccination and serious adverse events, except for migraine. Although a significant increase in risk was observed for migraine at the two year follow-up (adjusted rate ratio 1.10, $95 \%$ confidence interval 1.03 to 1.16 ), no significant increase was observed at 90 days (1.04, 0.85 to 1.26) and 180 days (1.09, 0.94 to 1.25) (supplementary section C).

Results from subgroup analyses for vaccine subtype were also consistent with those of the main findings. Although no significant increase was found for migraine in the bivalent HPV vaccinated group compared with HPV unvaccinated group (1.07, 0.96 to 1.20), the quadrivalent HPV vaccinated group showed a statistically significant increase in migraine compared with the HPV unvaccinated group (1.13, 1.03 to 1.24 ) (fig 4).

\section{Discussion}

In this nationwide study of girls aged 11-14 years who received HPV, Japanese encephalitis, and tetanus, diphtheria, and acellular pertussis vaccines in South Korea in 2017, we used two complementary study designs to investigate the association between HPV vaccination and serious adverse events. All 33 predefined outcomes except for migraine showed no association between HPV vaccination and serious adverse events based on more than 400000 girls and around 500000 vaccine doses, and these findings were consistent across the follow-up periods (90 days, 180 days, and 2 years). Subgroup analysis by HPV vaccine subtype also showed consistent results with our main findings.

In cohort analyses, of a total of 33 serious adverse events, a significant increase in risk was observed only for migraine (adjusted rate ratio $1.11,1.02$ to 


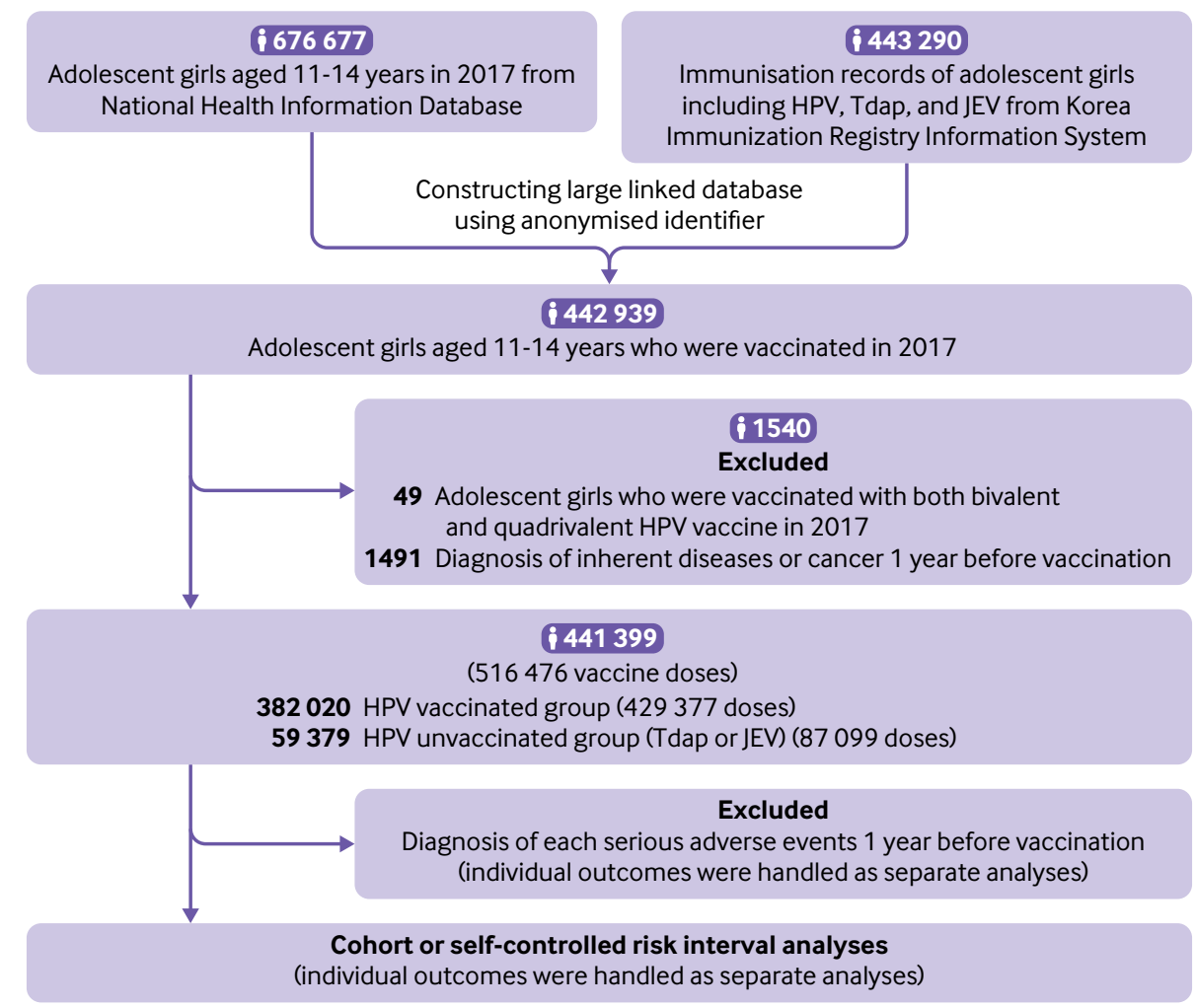

Fig 2 | Flowchart of enrolment using large database created by linking data from the Korea Immunization Registry Information System with data from the National Health Information Database. HPV=human papillomavirus; Tdap=tetanus, diphtheria, and acellular pertussis vaccine; JEV=Japanese encephalitis vaccine

1.22), but the point estimate and lower limit of the $95 \%$ confidence interval were close to 1 . Contrary to the results of the cohort analysis, the result of the self-controlled risk interval analysis presented no evidence of an association between HPV vaccination and migraine (relative risk 0.67, 95\% confidence interval 0.58 to 0.78 ), which did not support the risk of migraine after HPV vaccination. This also means that migraine occurs more frequently after the risk interval. Possible explanations could be outcome misclassification, validity of migraine diagnosis, and a relatively low number of girls with migraine compared

\begin{tabular}{|c|c|c|}
\hline Characteristics & HPV vaccinated group $(n=382020)$ & HPV unvaccinated group $(n=59379)$ \\
\hline Mean (SD) age at vaccination (years) & $12.42(0.82)$ & $11.84(0.56)$ \\
\hline \multicolumn{3}{|l|}{ Birth year: } \\
\hline 2003 & $108709(28.5)$ & $757(1.3)$ \\
\hline 2004 & $146094(38.2)$ & $15856(26.7)$ \\
\hline 2005 & $127217(33.3)$ & $42766(72.0)$ \\
\hline \multicolumn{3}{|l|}{ No of vaccine doses: } \\
\hline 1 & $148014(38.7)$ & NA \\
\hline 2 & $234006(61.3)$ & NA \\
\hline \multicolumn{3}{|l|}{ Region of residence: } \\
\hline Metropolitant & $185735(48.6)$ & $30830(51.9)$ \\
\hline Provincial or rural & $196285(51.4)$ & $28549(48.1)$ \\
\hline \multicolumn{3}{|l|}{ Types of health insurance: } \\
\hline Local & $81814(21.4)$ & $12645(21.3)$ \\
\hline Employee & $289744(75.8)$ & $45299(76.3)$ \\
\hline Medical aid & $10462(2.7)$ & $1435(2.4)$ \\
\hline \multicolumn{3}{|l|}{ Income levelt: } \\
\hline 1st quarter (most deprived) & $55438(14.5)$ & $7651(12.9)$ \\
\hline 2nd quarter & $48982(12.8)$ & $6968(11.7)$ \\
\hline 3rd quarter & $80956(21.2)$ & $12097(20.4)$ \\
\hline 4th quarter (most affluent) & $190635(49.9)$ & $31877(53.7)$ \\
\hline
\end{tabular}




\begin{tabular}{|c|c|c|c|c|c|c|}
\hline \multirow[b]{2}{*}{ Organ system disorders } & \multicolumn{3}{|c|}{ HPV vaccinated group } & \multicolumn{3}{|c|}{ HPV unvaccinated group } \\
\hline & $\begin{array}{l}\text { No of serious } \\
\text { adverse events }\end{array}$ & Person years & Incidence rate* $(95 \% \mathrm{Cl})$ & $\begin{array}{l}\text { No of serious } \\
\text { adverse events }\end{array}$ & Person years & Incidence rate* $(95 \% \mathrm{Cl})$ \\
\hline \multicolumn{7}{|l|}{ Endocrine diseases: } \\
\hline Graves' disease & 136 & 408038 & $33.3(28.2$ to 39.4$)$ & 20 & 60579 & $33.0(21.3$ to 51.2$)$ \\
\hline Hashimoto's thyroiditis & 215 & 407768 & 52.7 (46.1 to 60.3) & 22 & 60547 & 36.3 (23.9 to 55.2) \\
\hline Hyperthyroidism & 508 & 407603 & $124.6(114.3$ to 136.0$)$ & 61 & 60533 & 100.8 (78.4 to 129.5$)$ \\
\hline Hypothyroidism & 1227 & 406033 & 302.2 (285.7 to 319.6 ) & 138 & 60350 & 228.7 (193.5 to 270.2$)$ \\
\hline Type 1 diabetes & 82 & 408331 & 20.1 (16.2 to 24.9$)$ & 17 & 60623 & $28.0(17.4$ to 45.1$)$ \\
\hline \multicolumn{7}{|l|}{ Gastrointestinal diseases: } \\
\hline Crohn's disease & 83 & 408239 & 20.3 (16.4 to 25.2$)$ & 7 & 60615 & $11.6(5.5$ to 24.2$)$ \\
\hline Ulcerative colitis & 25 & 408310 & 6.1 (4.1 to 9.1$)$ & 4 & 60627 & $6.6(2.5$ to 17.6$)$ \\
\hline Peptic ulcer diseases & 6297 & 397433 & $1584.0(1546.0$ to 1624.0$)$ & 770 & 59422 & 1296.0 (1207.0 to 1391.0$)$ \\
\hline Pancreatitis & 902 & 406716 & 221.8 (207.8 to 236.7$)$ & 114 & 60400 & 188.7 (157.1 to 226.8) \\
\hline \multicolumn{7}{|l|}{ Cardiovascular diseases: } \\
\hline Raynaud's disease & 39 & 408327 & $9.6(7.0$ to 13.1$)$ & 3 & 60628 & $5.0(1.6$ to 15.3$)$ \\
\hline Venous thromboembolism & 26 & 408340 & $6.4(4.3$ to 9.4$)$ & 6 & 60628 & 9.9 (4.5 to 22.0$)$ \\
\hline Vasculitis & 161 & 408129 & 39.5 (33.8 to 46.0$)$ & 27 & 60605 & 44.6 (30.6 to 65.0$)$ \\
\hline Hypotension & 792 & 407401 & 194.4 (181.3 to 208.4$)$ & 87 & 60543 & 143.7 (116.5 to 177.3$)$ \\
\hline \multicolumn{7}{|c|}{ Musculoskeletal and systemic diseases: } \\
\hline Ankylosing spondylitis & 8 & 408361 & $2.0(1.0$ to 3.9$)$ & 0 & 60632 & $0.0(0.0$ to 0.0$)$ \\
\hline Behcet's syndrome & 27 & 408322 & $6.6(4.5$ to 9.6$)$ & 5 & 60627 & $8.3(3.4$ to 19.8$)$ \\
\hline Juvenile arthritis & 78 & 408181 & 19.1 (15.3 to 23.9) & 11 & 60601 & 18.2 (10.1 to 32.8$)$ \\
\hline Rheumatoid arthritis & 685 & 407378 & $168.1(156.0$ to 181.2$)$ & 88 & 60515 & $145.4(118.0$ to 179.2$)$ \\
\hline Systemic lupus erythematosus & 15 & 408340 & $3.7(2.2$ to 6.1$)$ & 0 & 60626 & $0.0(0.0$ to 0.0$)$ \\
\hline \multicolumn{7}{|l|}{ Haematological diseases: } \\
\hline $\begin{array}{l}\text { Idiopathic thrombocytopenic } \\
\text { purpura }\end{array}$ & 28 & 408292 & 6.9 (4.7 to 9.9) & 9 & 60615 & $14.9(7.7$ to 28.5$)$ \\
\hline Henoch-Schönlein's purpura & 246 & 407892 & $60.3(53.2$ to 68.3$)$ & 50 & 60534 & 82.6 (62.6 to 109.0) \\
\hline \multicolumn{7}{|l|}{ Dermatological diseases: } \\
\hline Erythema nodosum & 26 & 408332 & $6.4(4.3$ to 9.4$)$ & 0 & 60628 & $0.0(0.0$ to 0.0$)$ \\
\hline Psoriasis & 624 & 407071 & 153.3 (141.7 to 165.8$)$ & 94 & 60469 & 155.5 (127.0 to 190.3$)$ \\
\hline \multicolumn{7}{|l|}{ Neurological diseases: } \\
\hline Bell's palsy & 102 & 408196 & $25.0(20.6$ to 30.3$)$ & 17 & 60607 & 28.1 (17.4 to 45.1$)$ \\
\hline Epilepsy & 595 & 406345 & 146.4 (135.1 to 158.7$)$ & 74 & 60325 & $122.7(97.7$ to 154.1$)$ \\
\hline Narcolepsy & 33 & 408335 & $8.1(5.8$ to 11.4$)$ & 4 & 60628 & $6.6(2.5$ to 17.6$)$ \\
\hline Paralysis & 19 & 408345 & $4.7(3.0$ to 7.3$)$ & 4 & 60626 & $6.6(2.5$ to 17.6$)$ \\
\hline Migraine & 4949 & 400699 & $1235.0(1201.0$ to 1270.0$)$ & 551 & 59835 & $920.9(847.1$ to 1001.0$)$ \\
\hline Guillain-Barré syndrome & 5 & 408363 & $1.2(0.5$ to 2.9$)$ & 5 & 60626 & $8.3(3.4$ to 19.8$)$ \\
\hline Optical neuritis & 103 & 408211 & $25.2(20.8$ to 30.6$)$ & 9 & 60612 & $14.9(7.7$ to 28.5$)$ \\
\hline Neuralgia and neuritis & 431 & 407703 & 105.7 (96.2 to 116.2$)$ & 62 & 60555 & 102.4 (79.8 to 131.3$)$ \\
\hline Intracerebral haemorrhage & 38 & 408306 & $9.3(6.8$ to 12.8$)$ & 3 & 60622 & $5.0(1.6$ to 15.3$)$ \\
\hline $\begin{array}{l}\text { Extrapyramidal and movement } \\
\text { disorders }\end{array}$ & 215 & 408106 & 52.7 (46.1 to 60.2$)$ & 29 & 60594 & 47.9 (33.3 to 68.9) \\
\hline Tuberculosis & 110 & 408143 & $27.0(22.4$ to 32.5$)$ & 14 & 60612 & 23.1 (13.7 to 39.0) \\
\hline
\end{tabular}

with those in the general population. Above all, we suspected that this inconsistency would probably be attributed to age. From a literature review and clinical consultation, we identified that hormonal changes and menstruation are well known risk factors for migraine. ${ }^{35}$ Considering the overall mean age at menarche (12.7 years) in the Korean population, ${ }^{36}$ our study population might encounter menstrual related migraine during the follow-up period. Moreover, given the mean age differences between the HPV vaccinated (12.42 years) and HPV unvaccinated (11.84 years) groups, menstrual related migraine is likely to occur more often in the HPV vaccinated group, resulting in statistically significantly increased results. Along with the results of the cohort analysis, our sensitivity analyses support an increased effect of age on migraine over time. Although sensitivity analyses for the short term period did not show a significant increase in the risk of migraine (adjusted rate ratios: 90 days, $1.04,0.85$ to 1.26 ; 180 days $1.09,0.94$ to 1.25 ), significant results were found for the long term period (2 years, 1.10, 1.03 to 1.16). Therefore, the effect of age would possibly contribute to this result, but this finding should be interpreted with caution given the pathophysiology of migraine.

\section{Comparison with other studies}

Our findings of no significant increase in serious adverse events show the safety of HPV vaccination and corroborate the findings of previous studies. Several studies have found no association between HPV vaccination and autoimmune diseases, a major concern in vaccine safety studies. ${ }^{13} 141937$ Although a recent meta-analysis highlighted no clear association between HPV vaccines and autoimmune diseases, the study also suggested a significant increase in the risk 


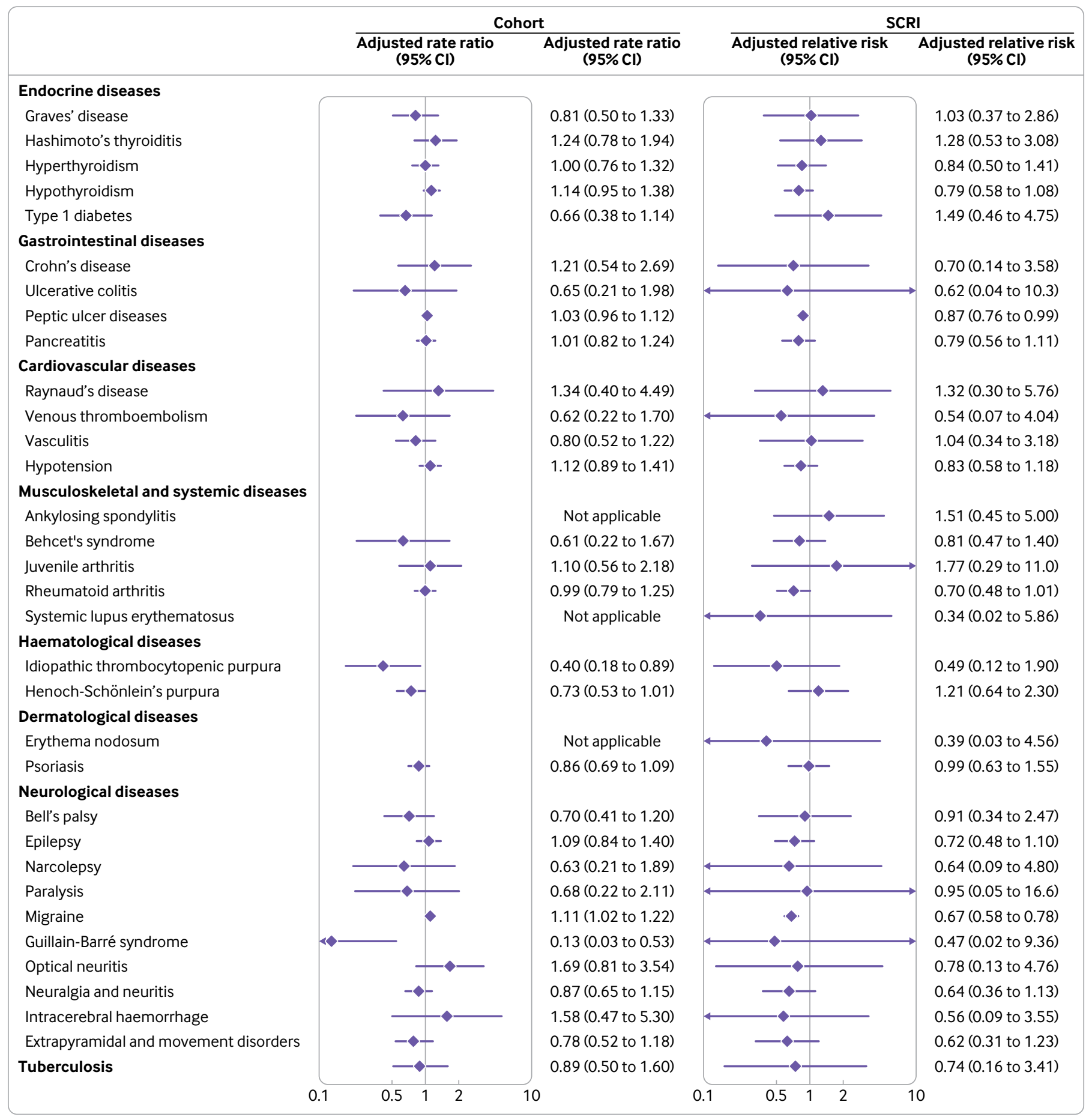

Fig 3 | Association between human papillomavirus vaccination and serious adverse events in both the cohort and the self-controlled risk interval (SCRI) analyses among girls aged 11-14 years and vaccinated in South Korea in 2017

of some types of autoimmune diseases: Hashimoto's thyroiditis (odds ratio 1.25 , 95\% confidence interval 1.09 to 1.44 ) and Raynaud's disease (1.63, 1.21 to 2.20). ${ }^{38}$ We speculated that this increased risk resulted from the unmasking phenomenon, which can occur when vaccinated groups are compared with unvaccinated groups. $^{22}$ Visiting a medical institution for vaccination offers an opportunity to assess symptoms that might not have been assessed otherwise. The fundamental difference between vaccinated and unvaccinated groups would bias the results towards an increased risk after vaccination. Thus we used comparator vaccines to account for the unmasking phenomenon, and thereby Hashimoto's thyroiditis showed no increased risk in both cohort and self-controlled risk interval analyses (adjusted rate ratio $1.24,95 \%$ confidence interval 0.78 to 1.94 ; adjusted relative risk 1.28 , 95\% confidence interval 


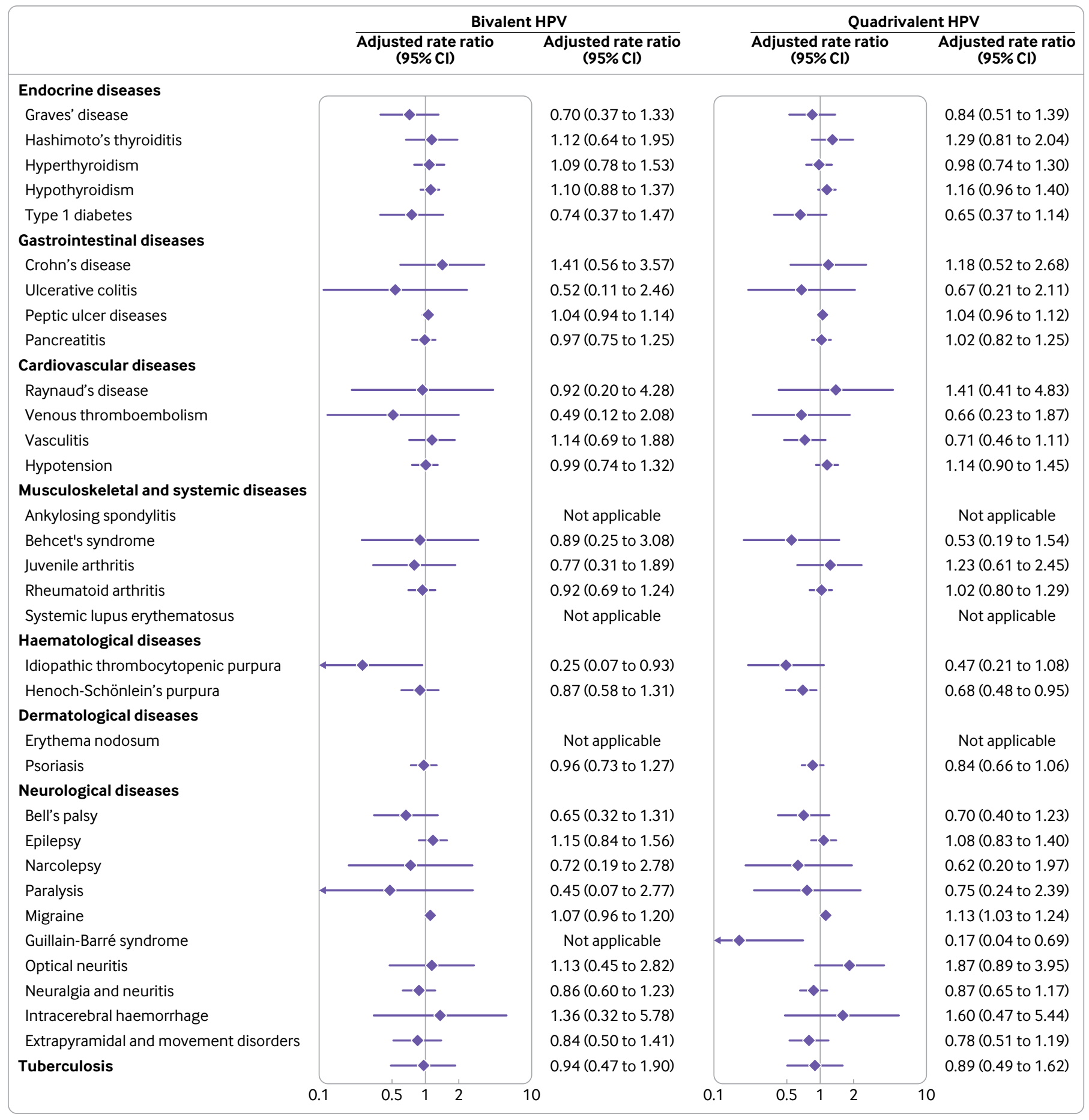

Fig 4 | Association between human papillomavirus vaccination and serious adverse events in subgroup analyses by vaccine subtypes among girls aged 11-14 years and vaccinated in South Korea in 2017

0.53 to 3.08 , respectively), and Raynaud's disease also showed no increased risk in the self-controlled risk interval analysis (adjusted relative risk 1.32, 95\% confidence interval 0.30 to 5.76; rate ratios could not be calculated in the cohort analysis because the disease was not observed in the HPV unvaccinated group).

Several studies have focused on the association between HPV vaccination and migraine, and one study based on a passive surveillance database suggested migraine is a safety signal for HPV vaccination. ${ }^{30} 39$ The studies did not confirm a clear relation between migraine and HPV vaccination, but further research is required to clarify the causal association behind the disease. A cohort study conducted in the Netherlands identified no significant increase in the risk of migraine after immunisation with the bivalent HPV vaccine. ${ }^{40}$ Although the study was limited by insufficient sample size and lack of power, this result was consistent with 
our result from subgroup analysis of the bivalent HPV vaccine (adjusted rate ratio 1.07, 95\% confidence interval 0.96 to 1.20 ).

\section{Strength and limitations of this study}

By linking two different nationwide databases-a vaccine registry and health insurance database-our study provided results for the entire population of South Korea. ${ }^{41}$ Using this nationwide linked database allowed us to detect associations between HPV vaccine and rare or serious diseases without losing statistical power. In addition, as the South Korean vaccine registry compiles all immunisation data under the national immunisation programme, including vaccines against HPV, Japanese encephalitis, and tetanus, diphtheria, and acellular pertussis, the omission of information on vaccine use would be rare. Unlike previous studies, we adopted two complementary analyses-cohort and self-controlled risk interval. The cohort study design allowed us to provide more generalisable evidence and has higher statistical power, and the self-controlled risk interval study design enabled us to adjust time invariant confounders, including socioeconomic status, underlying chronic or congenital diseases, use of medical resources, and other unmeasured confounders. ${ }^{42}$ Specifically, in the cohort analysis we assessed the risk of serious adverse events after HPV vaccination by comparing the HPV vaccinated group with a comparator vaccinated group rather than with an unvaccinated group. Since the unvaccinated group includes those who decline vaccination, their clinical and personal characteristics and health seeking behaviour are likely to be different from those who are vaccinated. ${ }^{43}$ Thus, by comparing relatively homogeneous populations our approach secures internal validity and can produce reliable results by controlling for the unmasking phenomenon and fundamental differences between vaccinated and unvaccinated groups. In addition, our study assessed several serious adverse events suspected from previous studies and contains acute and insidious diseases through consultation of the medical advisory committee on vaccine safety, which enabled us to provide comprehensive safety information on HPV vaccines. Through our subgroup analyses we were also able to supply more information on the safety of each HPV vaccine subtype because most previous research was on vaccination using the quadrivalent HPV vaccine.

Our study has some limitations. Firstly, our case definition relied on ICD-10 codes recorded in health insurance data. As the diagnosis records using ICD10 codes are not perfectly consistent with the medical records of patients, the discrepancy between health insurance data and medical records can overestimate or underestimate the real incidence of selected outcomes. ${ }^{445}$ However, in a validation study regarding the National Health Information Database, the overall positive predictive value of the diagnosis was $82 \%,{ }^{21}$ which enabled us to provide reliable information on vaccine safety. Secondly, because of limited access to medical records we used the date of diagnosis rather than the date of disease onset. The delay between disease onset and diagnosis would have contributed to the misclassification of cases if onset and diagnosis did not occur in the same risk period. This random misclassification bias would skew the results towards a null association. To resolve any delay from onset to diagnosis, we selected one year as the primary risk period and conducted sensitivity analyses with varying risk periods (90 days, 180 days, and 2 years). Thirdly, a possibility of misclassification about vaccine use remains. As both the bivalent and the quadrivalent HPV vaccines have been covered by funding from the South Korean government through the national immunisation programme, the registered vaccination records are complete, whereas vaccination records of a nonavalent HPV vaccine, which is not under the national immunisation programme, are not complete. A girl inoculated with a comparator vaccine and the nonavalent HPV vaccine is likely to be classified as being HPV unvaccinated. However, given the equivalent effect of the nonavalent and quadrivalent HPV vaccines, ${ }^{46}$ few girls are likely to be inoculated with the nonavalent HPV vaccine by paying additional costs. Fourthly, because many of the predefined outcomes are rare, statistical power can be limited for some outcomes in our analyses. Notwithstanding, as we used a nationwide linked database, a few events might indicate that the event is not likely to be relevant. Finally, as our study population comprised vaccinated adolescent girls inoculated with either the HPV vaccine or the comparator vaccine, the generalisability of the findings to the entire South Korean population could be limited. However, considering that the study population included more than $65 \%$ of all girls aged 11-14 years in South Korea, we believe that our findings are representative of the general population.

\section{Conclusion and policy implications}

From a public health perspective, HPV vaccination and screening were adapted worldwide as general strategies for the prevention of cervical cancer. ${ }^{47}$ Fear of possible adverse events is, however, one of the main determinants of HPV vaccine hesitancy and of undermining public trust in vaccines. 9489 The inevitable consequence of HPV vaccine hesitancy in Japan was estimated to result in around 5000 deaths due to cervical cancer during 2013-19. ${ }^{50}$ In light of the impact of HPV vaccine hesitancy on cervical cancer mortality, establishing the safety of HPV vaccines and alleviating public concerns is imperative. Our study evaluated the association between HPV vaccination and serious adverse events (those related to autoimmunity or other organ system disorders) to provide safety information on HPV vaccination in South Korea. Of 33 predefined serious adverse events in our study, we found no significant increase in risk for 32, and these findings were consistent across the self-controlled risk interval, sensitivity, and subgroup analyses. Although a slightly increased risk was observed for migraine in the cohort analysis, this should be interpreted with 
caution considering the pathophysiology of migraine and the population of interest. The results for safety profiles of the HPV vaccine in adolescent girls in South Korea are consistent with the evidence in Western populations.

We thank the Korea Disease Control and Prevention Agency for providing anonymous data from individual immunisation records, and the National Health Insurance Service for providing invaluable data.

Contributors: DY collected the data, performed statistical analyses, interpreted the data, and wrote the manuscript. JHL designed the overall research, interpreted the data, and contributed to the writing of the manuscript. HL collected the data and contributed to the statistical analyses and writing of the manuscript. JYS designed the study, supervised the statistical analyses and interpretation of data, and critically revised the manuscript. JYS, guarantor of the study, accepts full responsibility for the research, had access to the data, and controlled the decision to publish. The corresponding author attests that all listed authors meet authorship criteria and that no others meeting the criteria have been omitted.

Funding: This research was supported by Government-wide R\&D Fund project for infectious disease research (GFID), Republic of Korea (grant No HG18C0068). The funder was not involved in the study design; in the collection, analysis, and interpretation of data; in the writing of the report; and in the decision to submit the article for publication.

Competing interests: All authors have completed the ICMJE uniform disclosure form at www.icmje.org/coi disclosure.pdf and declare: all authors had financial support from government-wide research and development fund project for infectious disease research; I-YS received grants from the Ministry of Food and Drug Safety; the Ministry of Health and Welfare; the National Research Foundation of Korea; the Government-wide R\&D Fund project for infectious disease research; and pharmaceutical companies, including Amgen, Pfizer, Hoffmann-La Roche, Dong-A ST, and Yungjin; no other relationships or activities that could appear to have influenced the submitted work.

Ethical approval: This study was approved by the institutional review board of Sungkyunkwan University (No 2019-03-017), which waived the requirement for informed consent as only deidentified data were used in this study.

Data sharing: No additional data available.

The lead author (J-YS) affirms that this manuscript is an honest, accurate, and transparent account of the study being reported; that no important aspects of the study have been omitted; and that any discrepancies from the study as planned (and, if relevant, registered) have been explained.

Dissemination to participants and related patient and public communities: We have no plans to disseminate the results of the research to study participants or the relevant patient community. As anonymised data were used in this study, the authors had no direct contact information of the study participants to disseminate the results of the research.

Provenance and peer review: Not commissioned; externally peer reviewed.

This is an Open Access article distributed in accordance with the Creative Commons Attribution Non Commercial (CC BY-NC 4.0) license, which permits others to distribute, remix, adapt, build upon this work non-commercially, and license their derivative works on different terms, provided the original work is properly cited and the use is noncommercial. See: http://creativecommons.org/licenses/by-nc/4.0/.

1 de Martel C, Plummer M, Vignat J, Franceschi S. Worldwide burden of cancer attributable to HPV by site, country and HPV type. Int J Cancer 2017;141:664-70. doi:10.1002/ijc.30716

2 Ferlay J, Soerjomataram I, Dikshit R, et al. Cancer incidence and mortality worldwide: sources, methods and major patterns in GLOBOCAN 2012. Int J Cancer 2015;136:E359-86. doi:10.1002/ ijc. 29210

3 Markowitz LE, Tsu V, Deeks SL, et al. Human papillomavirus vaccine introduction--the first five years. Vaccine 2012;30(Suppl 5):F139-48. doi:10.1016/j.vaccine.2012.05.039

4 Global HPV vaccine introduction overview: projected and current national introductions, demonstration/pilot projects, gender-neutral vaccination programs, and global HPV vaccine introduction maps (2006-2022). 2019 https://path.azureedge.net/media/documents/ GlobalHPVVaccinelntroOverview_Nov2019.pdf (accessed 26 September 2020)
5 Schedules and strategies for human papillomavirus (HPV) immunization: World Health Organization; 2016 [updated 27 September 2016. https://www.who.int/immunization/ sage/meetings/2016/october/1_HPV_vaccine_background_ document_27Sept2016.pdf?ua=1

6 Kim MA, Han GH, Kim JH, Seo K. Current Status of Human Papillomavirus Infection and Introduction of Vaccination to the National Immunization Program in Korea: an Overview. J Korean Med Sci 2018:33:e331. doi:10.3346/jkms.2018.33.e331

7 National Immunization Program for children 2019 [updated 16 July 2019]. http://www.cdc.go.kr/contents.es?mid=a30301140000 (accessed 1 March 2020).

8 Recommendation of HPV immunization for adolescent girls born between 2006 to 2007 Korea Centers for Disease Control and Prevention, 2019. https://nip.cdc.go.kr/irgd/support.do? service $=$ getNewsView\&strNum $=252 \&$ PROSEQNUM $=429 \&$ SEARCHTYPE $=\&$ SEARCHWARD $=($ accessed 20 March 2020)

9 Bonanni P, Zanella B, Santomauro F, Lorini C, Bechini A, Boccalini S. Safety and perception: What are the greatest enemies of HPV vaccination programmes? Vaccine 2018;36:5424-9. doi:10.1016/j. vaccine.2017.05.071

10 Jørgensen L, Gøtzsche PC, Jefferson T. Benefits and harms of the human papillomavirus (HPV) vaccines: systematic review with metaanalyses of trial data from clinical study reports. Syst Rev 2020;9:43. doi:10.1186/s13643-019-0983-y

11 Survey of attitudes toward human papillomavirus vaccine vaccination 2017 https://www.cdc.go.kr/board/board. es? $\mathrm{mid}=\mathrm{a} 205010000000$ \&bid=0015\&act=view\&list no $=75836 \&$ tag $=\& n P a g e=112$ (accessed 4 June 2020)

12 Scheller NM, Svanström H, Pasternak B, et al. Quadrivalent HPV vaccination and risk of multiple sclerosis and other demyelinating diseases of the central nervous system. JAMA 2015;313:54-61. doi:10.1001/jama.2014.16946

13 Arnheim-Dahlström L, Pasternak B, Svanström H, Sparén P, Hviid A. Autoimmune, neurological, and venous thromboembolic adverse events after immunisation of adolescent girls with quadrivalent human papillomavirus vaccine in Denmark and Sweden: cohort study. BMJ 2013;347:f5906. doi:10.1136/bmj.f5906

14 Miranda S, Chaignot C, Collin C, Dray-Spira R, Weill A, Zureik $M$. Human papillomavirus vaccination and risk of autoimmune diseases: A large cohort study of over 2 million young girls in France. Vaccine 2017:35:4761-8. doi:10.1016/j.vaccine.2017.06.030

15 Hviid A, Thorsen NM, Valentiner-Branth P, Frisch M, Mølbak K Association between quadrivalent human papillomavirus vaccination and selected syndromes with autonomic dysfunction in Danish females: population based, self-controlled, case series analysis. BMJ 2020;370:m2930. doi:10.1136/bmj.m2930

16 Hviid A, Svanström H, Scheller NM, Grönlund O, Pasternak B, Arnheim-Dahlström L. Human papillomavirus vaccination of adult women and risk of autoimmune and neurological diseases. J Intern Med 2018;283:154-65. doi:10.1111/joim.12694

17 Grimaldi-Bensouda L, Guillemot D, Godeau B, et al, PGRx-AID Study Group. Autoimmune disorders and quadrivalent human papillomavirus vaccination of young female subjects. J Intern Med 2014;275:398-408. doi:10.1111/joim.12155

18 Yih WK, Greene SK, Zichittella L, et al. Evaluation of the risk of venous thromboembolism after quadrivalent human papillomavirus vaccination among US females. Vaccine 2016;34:172-8. doi:10.1016/j.vaccine.2015.09.087

19 Willame C, Rosillon D, Zima J, et al. Risk of new onset autoimmune disease in 9- to 25-year-old women exposed to human papillomavirus-16/18 AS04-adjuvanted vaccine in the United Kingdom. Hum Vaccin Immunother 2016;12:2862-71. doi:10.1080 /21645515.2016.1199308

20 Cheol Seong S, Kim YY, Khang YH, et al. Data Resource Profile: The National Health Information Database of the National Health Insurance Service in South Korea. Int J Epidemiol 2017;46:799-800. doi:10.1093/ije/dyw253

21 Park EJS, Jeon S, Lee S, Lee J, Choi D. Report of the evaluation for validity of discharged diagnoses in Korean Health Insurance database. Health Insurance Review and Assessment Service, 2017

22 Jacobsen SJ, Sy LS, Ackerson BK, et al. An unmasking phenomenon in an observational post-licensure safety study of adolescent girls and young women. Vaccine 2012;30:4585-7. doi:10.1016/j. vaccine.2012.04.103

23 Park HJ, Kim SI, Song R et al A 6-year Prospective Observational, Multi-Center Post-Marketing Surveillance of the Safety of Tetanus Toxoid, Reduced Diphtheria Toxoid, and Acellular Pertussis (Tdap) Vaccine in Korea. J Korean Med Sci 2019;34:e105. doi:10.3346/ jkms.2019.34.e105

24 Schuller E, Klingler A, Dubischar-Kastner K, Dewasthaly S, Müller Z. Safety profile of the Vero cell-derived Japanese encephalitis virus (JEV) vaccine IXIARO(®). Vaccine 2011;29:8669-76. doi:10.1016/j. vaccine.2011.08.117 
25 Chokephaibulkit K, Houillon G, Feroldi E, Bouckenooghe A. Safety and immunogenicity of a live attenuated Japanese encephalitis chimeric virus vaccine (IMOJEV $®)$ in children. Expert Rev Vaccines 2016;15:153-66. doi:10.1586/14760584.2016. 1123097

26 Kim CJ, Song R, Chen J, et al. Six-year multi-centre, observational, post-marketing surveillance of the safety of the HPV-16/18 AS04-adjuvanted vaccine in women aged 10-25 years in Korea. Pharmacoepidemiol Drug Saf 2017;26:837-42. doi:10.1002/ pds.4175

27 Markowitz LE, Hariri S, Unger ER, Saraiya M, Datta SD, Dunne EF. Post-licensure monitoring of HPV vaccine in the United States. Vaccine 2010;28:4731-7. doi:10.1016/j.vaccine.2010.02.019

28 Angelo MG, Zima J, Tavares Da Silva F, Baril L, Arellano F. Postlicensure safety surveillance for human papillomavirus-16/18AS04-adjuvanted vaccine: more than 4 years of experience. Pharmacoepidemiol Drug Saf 2014;23:456-65. doi:10.1002/ pds. 3593

29 Arana JE, Harrington T, Cano M, et al. Post-licensure safety monitoring of quadrivalent human papillomavirus vaccine in the Vaccine Adverse Event Reporting System (VAERS), 2009-2015. Vaccine 2018:36:1781-8 doi:10.1016/i.vaccine.2018.02.034

30 Ran J, Yang JY, Lee JH, Kim HJ, Choi JY, Shin JY. Signal detection of human papillomavirus vaccines using the Korea Adverse Events Reporting System database, between 2005 and 2016. Int J Clin Pharm 2019:41:1365-72 doi:10.1007/s11096-019-00881-9

31 Ozawa K, Hineno A, Kinoshita T, Ishihara S, Ikeda SI. Suspected Adverse Effects After Human Papillomavirus Vaccination: A Temporal Relationship Between Vaccine Administration and the Appearance of Symptoms in Japan. Drug Saf 2017;40:1219-29. doi:10.1007/ s40264-017-0574-6

32 Poddighe D, Castelli L, Marseglia GL, Bruni P. A sudden onset of a pseudo-neurological syndrome after HPV-16/18 AS04-adjuvated vaccine: might it be an autoimmune/inflammatory syndrome induced by adjuvants (ASIA) presenting as a somatoform disorder?/mmunol Res 2014:60:236-46. doi:10.1007/s12026-014-8575-3

33 Baker MA, Lieu TA, Li L, et al. A vaccine study design selection framework for the postlicensure rapid immunization safety monitoring program. Am J Epidemiol 2015;181:608-18. doi:10.1093/aje/kwu322

34 Chao C, Jacobsen SJ. Evaluation of autoimmune safety signal in observational vaccine safety studies. Hum Vaccin Immunother 2012;8:1302-4. doi:10.4161/hv.21268

35 Pavlović JM, Stewart WF, Bruce CA, et al. Burden of migraine related to menses: results from the AMPP study. J Headache Pain 2015;16:24. doi:10.1186/s10194-015-0503-y

36 Lee MH, Kim SH, Oh M, Lee KW, Park MJ. Age at menarche in Korean adolescents: trends and influencing factors. Reprod Health 2016;13:121. doi:10.1186/s12978-016-0240-y

37 Chao C, Klein NP, Velicer CM, et al. Surveillance of autoimmune conditions following routine use of quadrivalent human papillomavirus vaccine. I Intern Med 2012;271:193-203. doi:10.1111/j.1365-2796.2011.02467.x
38 Willame C, Gadroen K, Bramer W, Weibel D, Sturkenboom M Systematic Review and Meta-analysis of Postlicensure Observational Studies on Human Papillomavirus Vaccination and Autoimmune and Other Rare Adverse Events. Pediatr Infect Dis I 2020;39:287-93. doi:10.1097/INF.0000000000002569

39 Brinth L, Theibel AC, Pors K, Mehlsen J. Suspected side effects to the quadrivalent human papilloma vaccine. Dan Med J 2015;62:A5064.

40 Schurink-Van't Klooster TM, de Ridder MA, Kemmeren JM, et al. Examining a possible association between human papilloma virus (HPV) vaccination and migraine: results of a cohort study in the Netherlands. Eur J Pediatr 2015;174:641-9. doi:10.1007/s00431-014-2444-X

41 Kim L, Kim J-A, Kim S. A guide for the utilization of Health Insurance Review and Assessment Service National Patient Samples. Epidemiol Health 2014;36:e2014008-08. doi:10.4178/epih/e2014008

42 Li R, Stewart B, Weintraub E. Evaluating efficiency and statistical power of self-controlled case series and self-controlled risk interva designs in vaccine safety. J Biopharm Stat 2016;26:686-93. doi:10.1 080/10543406.2015.1052819

43 Smith PJ, Chu SY, Barker LE. Children who have received no vaccines: who are they and where do they live?Pediatrics 2004;114:187-95. doi:10.1542/peds.114.1.187

44 Lim S-S, Lee W, Kim Y-K, et al. The cumulative incidence and trends of rare diseases in South Korea: a nationwide study of the administrative data from the National Health Insurance Service database from 2011-2015. Orphanet / Rare Dis 2019;14:49. doi:10.1186/s13023-019-1032-6

45 O'Malley KJ, Cook KF, Price MD, Wildes KR, Hurdle JF, Ashton CM. Measuring diagnoses: ICD code accuracy. Health Serv Res 2005;40:1620-39. doi:10.1111/j.1475-6773.2005.00444.x

46 Bergman H, Buckley BS, Villanueva G, et al. Comparison of different human papillomavirus (HPV) vaccine types and dose schedules for prevention of HPV-related disease in females and males. Cochrane Database Syst Rev 2019;2019:CD013479. doi:10.1002/14651858.CD013479

47 Jit M, Brisson M, Portnoy A, Hutubessy R. Cost-effectiveness of female human papillomavirus vaccination in 179 countries: a PRIME modelling study. Lancet Glob Health 2014;2:e406-14. doi:10.1016/ S2214-109X(14)70237-2

48 Stretch R, Roberts SA, McCann R, et al. Parental attitudes and information needs in an adolescent HPV vaccination programme. $\mathrm{Br}$ J Cancer 2008;99:1908-11. doi:10.1038/sj.bjc.6604766

49 Egawa-Takata T, Ueda Y, Morimoto A, et al. Survey of Japanese mothers of daughters eligible for human papillomavirus vaccination on attitudes about media reports of adverse events and the suspension of governmental recommendation for vaccination. J Obstet Gynaecol Res 2015;41:1965-71. doi:10.1111/jog.12822

50 Simms KT, Hanley SJB, Smith MA, Keane A, Canfell K. Impact of HPV vaccine hesitancy on cervical cancer in Japan: a modelling study. Lancet Public Health 2020:5:e223-34 doi:10.1016/S24682667(20)30010-4

Supplementary information: sections A-C 